\title{
Demonstrating quantum algorithm acceleration with NMR quantum computer
}

\author{
Mikio Nakahara ${ }^{1}$, Yasushi Kondo ${ }^{1}$, Kazuya Hata $^{1}$ and Shogo Tanimura ${ }^{2}$ \\ ${ }^{1}$ Department of Physics, Kinki University, \\ Higashi-Osaka 577-8502, Japan \\ ${ }^{2}$ Graduate School of Engineering, Osaka City University \\ Sumiyoshi-ku, Osaka, 558-8585, Japan
}

(Dated: October 28, 2018)

\begin{abstract}
In general, a quantum circuit is constructed with elementary gates, such as one-qubit gates and CNOT gates. It is possible, however, to speed up the execution time of a given circuit by merging those elementary gates together into larger modules, such that the desired unitary matrix expressing the algorithm is directly implemented. We demonstrate this experimentally by taking the twoqubit Grover's algorithm implemented in NMR quantum computation, whose pseudopure state is generated by cyclic permutations of the state populations. This is the first exact time-optimal solution, to our knowledge, obtained for a self-contained quantum algorithm.
\end{abstract}

PACS numbers: 03.67.Lx, 82.56.Jn

Keywords: NMR quantum computer, time-optimal control, Grover's algorithm

\section{INTRODUCTION}

A quantum computer is expected to solve some of computationally hard problems for a conventional digital computer 1]. The realization of a practical quantum computer is, however, still challenging in many respects [2]. One of the obstacles to any realization is a phenomenon known as "decoherence". The number of gate operations is severely limited since a quantum state is vulnerable due to interactions with the surroundings. Many strategies to overcome internal and external decoherence have been proposed, such as (1) quantum error correcting codes 3] (2) decoherence-free subspaces 4] (3) holonomic quantum computation [5], among others. In a conventional design of a quantum circuit, the so-called elementary set of gates $[6,7,8]$ such as single-qubit $S U(2)$ rotations and CNOT gates, are utilized. This design is motivated by the universality theorem proved in Ref. [8]. Suppose we are to implement an $n$-qubit unitary matrix $U_{\text {target}}$. In the conventional implementation this matrix is decomposed into a product of $S U(2)$ matrices acting bewteen a pair of basis vectors. Then a CNOT gate transforms one of the basis vectors to a new vector, so that the pair of the vectors forms a subspace corresponding to a single qubit on which the $S U(2)$ matrix acts.

Quantum algorithm acceleration is a totally new approach to the decoherence issue. This principle is originally proposed in the context of the holonomic quantum computation [9, 10, 11] and of Josephson junction qubits [12, 13, 14]. What is required to implement is not individual elementary gates but rather the $n$-qubit matrix $U_{\text {target }} \in U\left(2^{n}\right)$ realizing the given quantum algorithm. This matrix is directly implemented by properly choosing the control parameters in the Hamiltonian. The variational principle tells us that the gate execution time reduces, in general, compared to the conventional construction since the conventional gate sequence belongs to the possible solutions in direct implementation.
The proposal 12, 13, 14] has been made for fictitious Josephson charge qubits, which are still beyond reach. It is the purpose of this paper to demonstrate the acceleration of a quantum algorithm using an NMR quantum computer at our hand. For this demonstration, we employ two-qubit Grover's search algorithm whose initial state $|00\rangle$ is generated as a pseudopure state by cyclic permutations of the state populations [15]. In this process, we need to prepare three different initial states using $S U(4)$ transformations acting on a thermal equilibrium ensemble. It is found that the optimized pulse sequence reduces the gate operation time to $25 \%$ of the conventional pulse sequence operation time in two ensembles while it remains unchanged in one ensemble, which is already optimized using the conventional pulse sequence.

The next section is devoted to the formalism of our approach. The Hamiltonian for the two-qubit molecule is introduced and the time-evolution operator is defined. Section III is the main part of this paper, where the exact solutions for Grover's algorithm are obtained. These solutions are experimentally verified with our NMR computer. Section IV is devoted to summary and discussion.

\section{TIME-OPTIMAL PATH IN NMR QUANTUM COMPUTATION}

In the present paper, we are concerned with an NMR quantum computer with a two-qubit heteronucleus molecule. To be more specific, we use Carbon-13 labeled chloroform as a computational resource throughout our theoretical and experimental analyses. The Hamiltonian of the molecule is

$$
\begin{aligned}
H(\gamma)= & -\omega_{11}\left[\cos \phi_{1}\left(\sigma_{x} \otimes I_{2} / 2\right)+\sin \phi_{1}\left(\sigma_{y} \otimes I_{2} / 2\right)\right] \\
& -\omega_{12}\left[\cos \phi_{2}\left(I_{2} \otimes \sigma_{x} / 2\right)+\sin \phi_{2}\left(I_{2} \otimes \sigma_{y} / 2\right)\right] \\
& +2 \pi J \sigma_{z} \otimes \sigma_{z} / 4
\end{aligned}
$$


in the rotating frame of each nucleus. Here $I_{2}$ is the unit matrix of order 2 while $\sigma_{k}$ is the $k$ th Pauli matrix. The parameter $\omega_{1 i}$ is the amplitude of the RF pulse for the $i$ th spin while $\phi_{i}$ is its phase. These four independent control parameters are collectively denoted as $\gamma$.

The time-evolution operator

$$
U[\gamma(t)]=\mathcal{T} \exp \left[-i \int_{0}^{T} H(\gamma(t)) d t\right]
$$

associated with the Hamiltonian (10) is a functional of $\gamma(t)$, where $\mathcal{T}$ is the time-ordered product and we employ the natural units in which $\hbar$ is set to unity. Note that $U[\gamma(t)] \in S U(4)$ since (11) is traceless. Suppose we want to implement a unitary gate $U_{\text {target }}$. Then our task is to find a function $\gamma(t)$ such that

$$
U[\gamma(t)]=U_{\text {target }} .
$$

The solution of this problem is highly nontrivial and we often resort to numerical analysis 9, 10, 13, 14]. Note, moreover, that we would like to construct time-optimal solutions among many possible solutions of Eq. (3) to fight against decoherence. It must also be examined whether the optimal solution thus obtained has control parameters that are experimentally accessible.

It was shown in Refs. 16, 17] that there exists a geometrical picture for the time-optimal solution in the case of NMR quantum computation for two-qubit heteronuclear molecules. Here we summarize briefly the relevant aspects of their idea that are required for our investigation. The crucial observation is that the one-qubit rotation is carried out in a negligible time compared to that required for two-qubit operation generated by the $J$ coupling term in Eq. (11) and hence time required for the one-qubit rotation can be neglected in evaluating the gate operation time. Then it is natural to consider the homogeneous space $S U(4) / K$, where $K \equiv S U(2) \otimes S U(2)$, in which two-qubit gates that differ by one-qubit operations $U_{1} \otimes U_{2} \in K$ are identified. The path in the homogeneous space is then generated by the $J$-coupling term in Eq. (11). A remark is in order. The Hamiltonian (1) contains neither $\sigma_{z} \otimes I_{2}$ nor $I_{2} \otimes \sigma_{z}$ and it seems impossible to consider the equivalence class based on the whole subgroup $K$. Note, however, that these terms are easily generated by $\sigma_{x}$ and $\sigma_{y}$ as

$$
e^{-i \alpha \sigma_{z} / 2}=e^{i \pi \sigma_{y} / 4} e^{-i \alpha \sigma_{x} / 2} e^{-i \pi \sigma_{y} / 4},
$$

for example, and hence it makes sense to identify all the elements of $S U(4)$, that differ with each other modulo the subgroup $K$. With the same token, the terms $\sigma_{x} \otimes \sigma_{x}$ and $\sigma_{y} \otimes \sigma_{y}$ may be included in the $J$-coupling part of the Hamiltonian since

$$
\begin{aligned}
& e^{-i \pi J t \sigma_{x} \otimes \sigma_{x}}=e^{i \pi\left(\sigma_{y} \otimes I_{2}+I_{2} \otimes \sigma_{y}\right) / 4} \\
& \quad \times e^{-i \pi J t \sigma_{z} \otimes \sigma_{z} / 2} e^{-i \pi\left(\sigma_{y} \otimes I_{2}+I_{2} \otimes \sigma_{y}\right) / 4}
\end{aligned}
$$

for example. It should be noted that the set $\left\{\sigma_{x} \otimes \sigma_{x}, \sigma_{y} \otimes\right.$ $\left.\sigma_{y}, \sigma_{z} \otimes \sigma_{z}\right\}$ comprises the Cartan subalgebra of $S U(4)$ in a relevant basis and hence

$$
\begin{aligned}
& e^{-i\left(\alpha_{1} \sigma_{x} \otimes \sigma_{x}+\alpha_{2} \sigma_{y} \otimes \sigma_{y}+\alpha_{3} \sigma_{z} \otimes \sigma_{z}\right)} \\
& =e^{-i \alpha_{1} \sigma_{x} \otimes \sigma_{x}} e^{-i \alpha_{2} \sigma_{y} \otimes \sigma_{y}} e^{-i \alpha_{3} \sigma_{z} \otimes \sigma_{z}} .
\end{aligned}
$$

Suppose we want to find a time-optimal path $\gamma(t)$ connecting the unit matrix $I_{4}$ of order four and the target matrix $U_{\text {target }} \in S U(4)$. The prescription given above suggests that we should find the time-optimal path in $S U(4) / K$ that connects the equivalence classes $K$ and $U_{\text {target }} K$. In other words, we have to solve the matrix equation

$$
U_{\text {target }}=K_{2} U_{J}(t) K_{1},
$$

where $K_{1}, K_{2} \in K$ and

$$
U_{J}\left(t_{i}\right)=e^{-(i \pi J / 2)\left(t_{1} \sigma_{x} \otimes \sigma_{x}+t_{2} \sigma_{y} \otimes \sigma_{y}+t_{3} \sigma_{z} \otimes \sigma_{z}\right)} .
$$

The total execution time is then given by $T=\sum_{i=1}^{3} t_{i}$. The group $S U(4)$ is 15-dimensional, while each of the $K_{i}$ has six parameters and $U_{J}(t)$ has three, thus $2 \times 6+3=15$ parameters in total. It follows from Eqs. (4) and (5) that the Hamiltonian (11) contains the necessary and sufficient number of control parameters to construct an arbitrary quantum gate.

\section{TIME OPTIMAL SOLUTIONS OF GROVER'S ALGORITHM}

\section{A. Grover's Algorithm}

The solution of the decomposition (6) is difficult to find and we have to resort to numerical methods in general. As an example, we work out in the present section Grover's seach algorithm [18] in the framework outlined in the previous section. Grover's algorithm for a twoqubit case is implemented by unitary matrices of the form

$$
\begin{aligned}
U_{00} & =\left(\begin{array}{cccc}
-1 & 0 & 0 & 0 \\
0 & 0 & 1 & 0 \\
0 & 1 & 0 & 0 \\
0 & 0 & 0 & 1
\end{array}\right) \\
U_{01} & =\left(\begin{array}{cccc}
0 & 0 & 1 & 0 \\
-1 & 0 & 0 & 0 \\
0 & 0 & 0 & -1 \\
0 & -1 & 0 & 0
\end{array}\right) \\
U_{10} & =\left(\begin{array}{cccc}
0 & 1 & 0 & 0 \\
0 & 0 & 0 & -1 \\
-1 & 0 & 0 & 0 \\
0 & 0 & -1 & 0
\end{array}\right) \\
U_{11} & =\left(\begin{array}{cccc}
0 & 0 & 0 & 1 \\
0 & -1 & 0 & 0 \\
0 & 0 & -1 & 0 \\
-1 & 0 & 0 & 0
\end{array}\right)
\end{aligned}
$$

where $U_{i j}$ picks out the desired "data" $(i j)$ by operating on $|00\rangle$ as

$$
U_{i j}|00\rangle=e^{i \alpha}|i j\rangle, \quad(i, j=0,1)
$$


where we have written explicitly the possible phase on the rhs [21].

An interesting observation is that all the matrix elements are either 0 or \pm 1 , which implies that $U_{i j}$ is not only in $S U(4)$ but also in $S O(4)$. This further restricts the degrees of freedom of the matrix since $S O(4)$ is sixdimensional. In fact, our numerical results show that the decomposition (6) takes a highly constrained form.

\section{B. Optimization}

According to the presecription given in the previous section, we search a pulse sequence of the form $U_{i j}=$ $K_{2} U_{J} K_{1}$. Instead of solving this inverse problem, we define the equivalent variational problem which is easy to solve, at least numerically. Let

$$
U[\gamma(t)]=K_{2}\left(\gamma_{2}\right) U_{J}(t) K_{1}\left(\gamma_{1}\right)
$$

where

$$
K_{i}\left(\gamma_{i}\right)=e^{i\left(a_{1} \sigma_{x}+b_{1} \sigma_{y}+c_{1} \sigma_{z}\right)} \otimes e^{i\left(a_{2} \sigma_{x}+b_{2} \sigma_{y}+c_{2} \sigma_{z}\right)}
$$

and $U_{J}(t)$ has been given in Eq. (7). Now we define the penalty function $p[\gamma(t)]$ as

$$
p[\gamma(t)]=\left\|U[\gamma(t)]-U_{\text {target }}\right\|_{\mathrm{F}}
$$

where $\|A\|_{\mathrm{F}}=\sqrt{\operatorname{tr} A^{\dagger} A}$ is the Frobenius norm of a matrix $A$. Note that $p[\gamma(t)]$ is positive definite and that its zeros are the absolute minima. Thus solving the inverse problem is recast into a variational task for finding the absolute minima of $p[\gamma(t)]$.

To find the absolute minima, we have generated 512 initial conditions and searched for the optimal solutions with the polytope algorithm on a parallel computer with 512 CPUs. To our surprise, the execution time $T=$ $\sum_{i=1}^{3} t_{i}$ is discrete and assumes the values

$$
T=(n+1) / J, \quad n=0,1,2 \ldots
$$

for all four cases. The ambiguity $2 \pi n$ corresponds to the path leaving from $I_{4}$ and traversing the compact group $S U(4) n$ times before hitting the destination $U_{\text {target }} K_{2}$. The result shows that Fig. 1 of 16 is misleading at least in the present case: the distance between the cosets $I_{4} K_{1}$ and $U_{\text {target }} K_{2}$ is unique in the sense that the only degree of freedom left is how many times the path traverses $S U(4)$ before arriving at the target.

The optimal execution time obtained here, however, is the same as that for the conventional pulse sequence 15] in all cases. In other words, the conventional pulse sequences are already time-optimal. For such a simple algorithm, time-optimization may be carried out by inspection by experts. Therefore, we look for more complicated cases to demonstrate the power of this method. Suppose we would like to execute Grover's search algorithm with a room-temperature liquid state NMR computer. The sample is in a thermal equilibrium state and we will use the temporal averaging by cyclic permutations of state populations to obtain a pseudopure initial state. This is carried out by applying the unitary operators

$$
U_{\mathrm{cp}}=\left(\begin{array}{cccc}
1 & 0 & 0 & 0 \\
0 & 0 & 0 & 1 \\
0 & 1 & 0 & 0 \\
0 & 0 & 1 & 0
\end{array}\right)=\mathrm{CNOT}_{12} \mathrm{CNOT}_{21}
$$

and

$$
U_{\mathrm{cp}}^{2}=\left(\begin{array}{cccc}
1 & 0 & 0 & 0 \\
0 & 0 & 1 & 0 \\
0 & 0 & 0 & 1 \\
0 & 1 & 0 & 0
\end{array}\right)=\mathrm{CNOT}_{21} \mathrm{CNOT}_{12}
$$

to the initial thermal state before $U_{i j}$ is executed. Here $\mathrm{CNOT}_{i j}$ stands for the CNOT gate with the control bit $i$ and the target bit $j$.

We now present our search result for $U_{10}, U_{10} U_{\mathrm{cp}}$ and $U_{10} U_{\mathrm{cp}}^{2}$, the other $i j$ producing similar results.

- $U_{10}$ : An example of a typical time-optimal solution for $U_{10}$ is

$$
\begin{aligned}
& K_{1}=I_{2} \otimes I_{2} \\
& U_{J}=e^{i(\pi / 4)\left(\sigma_{x} \otimes \sigma_{x}-\sigma_{y} \otimes \sigma_{y}\right)} \\
& K_{2}=e^{-i(\pi / 4) \sigma_{z}} \otimes e^{i(\pi / 2 \sqrt{2})\left(\sigma_{x}+\sigma_{y}\right)} .
\end{aligned}
$$

The execution time is $T=1 / J$, which simply reproduces that for the conventional pulse sequence 15]. Therefore, the conventional pulse sequence is already optimized. We will employ the conventional pulse sequence for $U_{10}$ in the following.

- $U_{10} U_{\mathrm{cp}}$ : For this case, an example of the timeoptimal pulse sequence is

$$
\begin{aligned}
K_{1} & =I_{2} \otimes e^{-i(\pi / 4) \sigma_{x}} \\
U_{J} & =e^{-i(\pi / 4) \sigma_{z} \otimes \sigma_{z}} \\
K_{2} & =e^{i(\pi / 2) \sigma_{y}} \otimes e^{i(\pi / 3 \sqrt{3})\left(\sigma_{x}+\sigma_{y}+\sigma_{z}\right)} \\
& =e^{i(\pi / 2) \sigma_{y}} \otimes\left[e^{i(\pi / 4) \sigma_{y}} e^{i(\pi / 4) \sigma_{x}}\right],
\end{aligned}
$$

where the second line of $K_{2}$ shows the pulse sequence made of the existing terms in the Hamiltonian. It should be noted that this pulse sequence requires the execution time $T=1 / 2 J$ in spite of an additional gate $U_{\mathrm{cp}}$, which is composed of two CNOT gates and costs $1 / J$ of time to execute in the conventional pulse sequence. The execution time of the time-optimal pulse sequence is $25 \%$ of that for the conventional pulse sequence. The execution time for the other solutions takes discrete values $(n+1 / 2) / J,(n=0,1,2, \ldots)$. This corresponds to a path traversing $S U(4) n$ times before arriving at the $U_{\text {target }}$. 
- $U_{10} U_{\mathrm{cp}}^{2}$ : An example of the time-optimal pulse sequence is

$$
\begin{aligned}
K_{1} & =e^{-i(\pi / 3 \sqrt{3})\left(\sigma_{x}+\sigma_{y}+\sigma_{z}\right)} \otimes I_{2} \\
& =\left[e^{-i(\pi / 4) \sigma_{x}} e^{-i(\pi / 4) \sigma_{y}}\right] \otimes I_{2} \\
U_{J} & =e^{-i(\pi / 4) \sigma_{z} \otimes \sigma_{z}} \\
K_{2} & =e^{i(\pi / 4) \sigma_{x}} \otimes I_{2} .
\end{aligned}
$$

Note again that the execution time of the gate for the time-optimal pulse sequence is $25 \%$ of that for the conventional pulse sequence. In general, the execution time is $(n+1 / 2) / J,(n=0,1,2, \ldots)$ as in the above case.

The generators in the pulse sequences, that do not exist in the Hamiltonian (1), are rewritten in favor of the existing terms by making use of the conjugate transformations (4) and (5). The results are summarized in Table I. Also shown in the Table are the results according to the conventional pulse sequence for the respective gate.

\section{Experiments}

In our experiments, we used 0.6 milliliter, 200 millimolar sample of Carbon-13 labeled chloroform (Cambridge Isotopes) in d-6 acetone 15. Data were taken at room temperature with a JEOL ECA-500 (the hydrogen Larmor frequency being approximately $500 \mathrm{MHz}$ ) spectorometer [20]. The measured coupling strength is $J=215.5 \mathrm{~Hz}$ and the transverse relaxation time $T_{2}$ is $\sim 7.5 \mathrm{~s}$ for the hydrogen nucleus while $\sim 0.30 \mathrm{~s}$ for the carbon nucleus. The longitudinal relaxation time $T_{1}$ is measured to be $\sim 20 \mathrm{~s}$ for both nuclei. The spin 1 and 2 in Table $\llbracket$ correspond to Carbon-13 and H, respectively.

Our experimental results are shown in Fig. 11 which shows the spectra corresponding to the $|10\rangle$ state. The spectra in Fig. 1(a) were obtained by using hard pulses whose duration is $25 \mu \mathrm{s}$ for $\pi / 2$ pulses. We have intentionally introduced longer pulses with $250 \mu$ s duration to see the effect of imperfections, see Fig. 1(b). In the first case, we can well ignore the time evolution due to the J-coupling while pulses are applied since the characterist time for the $J$-coupling is $\sim 1 / J \sim 5 \mathrm{~ms}$. In the latter case, however, six $250 \mu$ s pulses amounts to the total duration of $1.5 \mathrm{~ms}$, which is comparable to $1 / J$ and we expect that the difference in the number of pulses will manifest. Figure 1(b) clearly demonstrates that time-optimal pulse sequences produce sharper main peak compared to the conventional pulse sequences and less unwanted signal, showing the superiority of our solutions. We also expect that quantum algorithm accreleration should be effective to fight against decoherence.

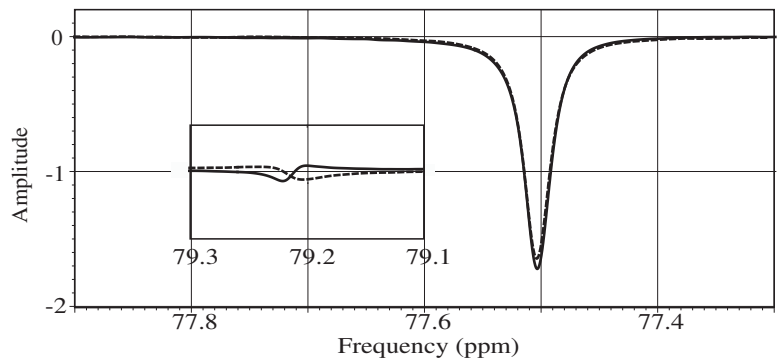

(b)

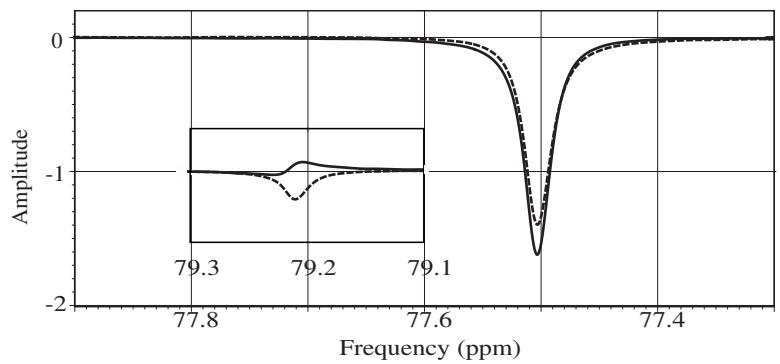

(a)

FIG. 1: The spectra for the peak corresponding to $|10\rangle$ state. Negative signal amplitudes indicate that the carbon nucleus is in the state $|1\rangle$, while appearance of the main signal at the frequency of $77.5 \mathrm{ppm}$ (instead of $79.2 \mathrm{ppm}$ ) implies that the hydrogen nucleus is in the $|0\rangle$ state. The insets show the signals in the vicinity of $79.2 \mathrm{ppm}$ where a signal may appear if the hydrogen nulceus has $|1\rangle$ component. The scales in the insets are the same as in the main panels. (a) The upper panel shows the spectra obtained with conventional (dotted line) and optimized (solid line) pulse sequences. Each $\pi / 2$ pulse duration is set to $25 \mu \mathrm{s}$. The main peak produced by the optimized pulse sequences is slightly sharper than that of the conventional one. (b) The lower panel shows spectra with conventional (dotted line) and optimized (solid line) pulse sequences, in which the duration of the $\pi / 2$-pulse is now set to $250 \mu \mathrm{s}$. The signal produced by the optimized pulse sequences is clearly better than that by the conventional ones. Note also that unwanted signal in the inset is weaker for the time-optimal pulse sequences.

\section{CONCLUSIONS AND DISCUSSION}

In summary, we have demonstrated both theoretically and experimentally that quantum algorithms may be accelerated if the unitary matrix realizing an algorithm is directly implemented by manipulating the control parameters in the Hamiltonian. We have verified this by implementing Grover's algorithm which picks out the "file" $|10\rangle$ starting from the pseudopure state generated by cyclic permutations of the state populations. We obtained the time-optimal pulse sequences and compared the results with those obtained by the conventional pulse sequences. It turns out that the gate $U_{10}$ is already optimized in the conventional pulse sequence while the gates $U_{10} U_{\mathrm{cp}}$ and $U_{10} U_{\mathrm{cp}}^{2}$ required for the cyclic permutations are accelerated so that the execution time is $25 \%$ of that for the conventional pulse sequence in both cases. The 


\begin{tabular}{|c|c|c|}
\hline \multicolumn{3}{|c|}{ Conventional pulse sequence } \\
\hline Gate & Pulse sequence & Execution time \\
\hline$\overline{U_{10}}$ & $\begin{array}{l}-\mathrm{Y}-(1 / 2 \mathrm{~J})-\mathrm{Ym}-\mathrm{Xm}-(1 / 2 \mathrm{~J})-\mathrm{Ym}-\mathrm{Xm}^{-} \\
-\mathrm{Y}-(1 / 2 \mathrm{~J})-\mathrm{Ym}-\mathrm{X}-(1 / 2 \mathrm{~J})-\mathrm{Ym}-\mathrm{Xm}-\end{array}$ & $1 / \mathrm{J}$ \\
\hline$U_{10} U_{\mathrm{cp}}$ & 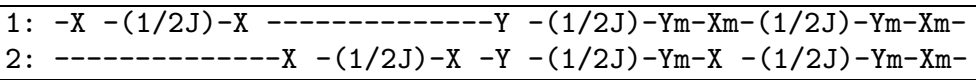 & $2 / \mathrm{J}$ \\
\hline$U_{10} U_{\mathrm{cp}}^{2}$ & $\begin{array}{ll}1: & -----1 \\
2: & -\mathrm{X}-(1 / 2 \mathrm{~J})-\mathrm{X}-\mathrm{X}-(1 / 2 \mathrm{~J})-\mathrm{X}-\mathrm{Y}-(1 / 2 \mathrm{~J})-\mathrm{Ym}-\mathrm{Xm}-(1 / 2 \mathrm{~J})-\mathrm{Ym}-\mathrm{Xm}- \\
-\end{array}$ & $2 / \mathrm{J}$ \\
\hline \multicolumn{3}{|c|}{ Time-optimal pulse sequence } \\
\hline Gate & Pulse sequence & Execution time \\
\hline$\overline{U_{10}}$ & $\begin{array}{l}-\mathrm{X}-(1 / 2 \mathrm{~J})-\mathrm{Xm}-\mathrm{Ym}-(1 / 2 \mathrm{~J})-\mathrm{Y}-\mathrm{Pi}(45)- \\
-\mathrm{X}-(1 / 2 \mathrm{~J})-\mathrm{Xm}-\mathrm{Y}-(1 / 2 \mathrm{~J})-\mathrm{X}-\mathrm{Ym} \quad-\end{array}$ & $1 / \mathrm{J}$ \\
\hline$U_{10} U_{\mathrm{cp}}$ & $\begin{array}{r}-\mathrm{X}-(1 / 2 \mathrm{~J})-\mathrm{Xm}-\mathrm{Ym}- \\
-\mathrm{Ym}-\mathrm{Ym}-\end{array}$ & $1 / 2 \mathrm{~J}$ \\
\hline$U_{10} U_{\mathrm{cp}}^{2}$ & $-\mathrm{Y}-\mathrm{X}-(1 / 2 \mathrm{~J})-\mathrm{Xm}-$ & $1 / 2 \mathrm{~J}$ \\
\hline
\end{tabular}

TABLE I: Control pulse sequences for Grover's algorithm, which picks out the 'file' $|10\rangle$ starting from the pseudopure state $|00\rangle$, which is obtained by cyclic permutations. The carbon nucleus is the first qubit while the hydrogen nucleus is the second. The upper Table shows the conventional pulse sequences [15] required to execute Grover's algorithm with the pseudopure state. The uppermost row shows the pulse sequence for the gate $U_{10}$, while the second and the third rows show those for $U_{10} U_{\mathrm{cp}}$ and $U_{10} U_{\mathrm{cp}}^{2}$, respectively. Here $\mathrm{X}(\mathrm{Xm})$ and $\mathrm{Y}(\mathrm{Ym})$ denote $\pi / 2$ pulse along $x(-x)$ and $y(-y)$ axis, respectively. The lower Table shows the time-optimal pulse sequences. The symbol Pi (45) denotes $\pi$-pulse along $(1,1,0)$ direction of the Bloch sphere.

number of the pulses required for $U_{10} U_{\mathrm{cp}}\left({ }_{10} U_{\mathrm{cp}}^{2}\right)$ is 4 (3) in the time-optimal pulses sequence, while it is 14 in both cases if the conventional pulse sequences are employed. The smallness in the number of pulses required leads to a higher-quality spectrum.

\section{Acknowledgements}

We would like to thank Manabu Ishifune for sample preparation, Toshie Minematsu for assistance in NMR operations and Katsuo Asakura and Naoyuki Fujii of JEOL for assistance in NMR pulse programming. Par- allel computing for the present work has been carried out with the CP-PACS computer under the "Large-scale Numerical Simulation Program" of Center for Computational Physics, University of Tsukuba. We would like to thank Martti Salomaa for drawing their attention to Refs. 16, 17] and careful reading of the manuscript. $\mathrm{MN}$ is grateful for partial support of a Grants-in-Aid for Scientific Research from Ministry of Education, Culture, Sports, Science and Technology (No. 13135215) and Japan Society for Promotion of Science (JSPS) (No. 14540346). ST would like to thank JSPS for partial support (No. 15540277).
[1] M. A. Nielsen, and I. L. Chuang, Quantum Computation and Quantum Information, (Cambridge University Press, Cambridge, 2000).

[2] F. De. Martini and C. Monroe (eds.), Experimental Quantum Computation and Information, (IOS Press, Amsterdam, 2002).

[3] A. R. Calderbank and P. W. Shor, Phys. Rev. A 54, 1098 (1996).

[4] D. A. Lidar, I. L. Chuang, and K. B. Whaley, Phys. Rev. Lett. 81, 2594 (1998).

[5] P. Zanardi and M. Rasetti, Pjys. Lett. A, 264, 94 (1999).

[6] J. J. Vartiainen, M. Möttönen, and M. M. Salomaa, Phys. Rev. Lett. 92, 177902 (2004).

[7] M. Möttönen, J. J. Vartiainen, V. Bergholm, and M. M. Salomaa, quant-ph/0404089 (2004).

[8] A. Barenco, C. H. Bennett, R. Cleve, D. P. DiVincenzo, N. Margolus, P. Shor, T. Sleator, J. A. Smolin, and H. Weinfurter, Phys. Rev. A 52, 3457 (1995).

[9] A. O. Niskanen, M. Nakahara, and M. M. Salomaa, Quantum Inf. Comput. 2, 560 (2002).
[10] A. O. Niskanen, M. Nakahara, and M. M. Salomaa, Phys. Rev. A 67, 012319 (2003).

[11] S. Tanimura, D. Hayashi, and M. Nakahara, Phys. Lett. A 325, 199 (2004).

[12] A. O. Niskanen, J. J. Vartiainen, and M. M. Salomaa, Phys. Rev. Lett. 90, 197901 (2003).

[13] J. V. Vartiainen, A. O. Niskanen, M. Nakahara and M. M. Salomaa, Int. J. Quant. Inf. 2, 1 (2004)

[14] J. V. Vartiainen, A. O. Niskanen, M. Nakahara and M. M. Salomaa, Phys. Rev. A, to be published.

[15] I. L. Chuang, N. Gershenfeld, and M. Kubinec, Phys. Rev. Lett. 80, 3408 (1998).

[16] N. Khaneja, R. Brockett, and S. J. Glaser, Phys. Rev. A 63, 032308 (2001).

[17] N. Khaneja, Harvard Thesis (2000).

[18] L. Grover, in Proceedings of the 28th Annual ACM Symposium on the Theory of Computation (ACM Press, New York, 1996), 212,

[19] L. K. Grover, Phys. Rev. Lett. 79, 325 (1997).

[20] http://www.jeol.com/nmr/nmr.html 
[21] The convensional pulse sequences [15] produce the matrices which differ from [8] - [11] in the phase of the matrix elements, which does not bring about any observable difference. 